\title{
Prognostic significance of preoperative mean platelet volume in resected non-small-cell lung cancer
}

\author{
SHOGO KUMAGAI ${ }^{1}$, JUNKO TOKUNO ${ }^{1}$, YUICHIRO UEDA ${ }^{1}$, SATOSHI MARUMO $^{1}$, TSUYOSHI SHOJI ${ }^{1}$, \\ TAKAFUMI NISHIMURA ${ }^{2}$, MOTONARI FUKUI ${ }^{1}$ and CHENG-LONG HUANG ${ }^{1}$ \\ ${ }^{1}$ Respiratory Disease Center, ${ }^{2}$ Department of Medical Oncology, Tazuke Kofukai \\ Medical Research Institute, Kitano Hospital, Osaka 530-8480, Japan
}

Received May 13, 2014; Accepted July 18, 2014

DOI: $10.3892 / \mathrm{mco} .2014 .436$

\begin{abstract}
An increased mean platelet volume (MPV) is an early marker of platelet activation. MPV was also shown to be associated with the pathophysiological characteristics of various types of cancer. A previous study demonstrated that MPV was significantly associated with the overall survival (OS) of patients with advanced non-small-cell lung cancer (NSCLC). However, there has been no analysis of the prognostic effect of MPV on patients with resected NSCLC. The aim of this study was to evaluate the contribution of MPV to the survival of patients with completely resected NSCLC. We retrospectively analyzed 308 consecutive patients with NSCLC who underwent curative resection at Kitano Hospital. The associations between MPV and clinicopathological factors were assessed. We also evaluated the effect of MPV on survival, using the two-tailed log-rank test and the Cox proportional hazards model. A MPV value of $8.50 \mathrm{fl}$ was considered to be the optimal cut-off value for prognosis. A low MPV was not associated with any other clinicopathological factors. The two-tailed log-rank test demonstrated that patients with a low MPV experienced a shorter disease-free survival (DFS) and overall survival (OS) $(\mathrm{P}=0.011$ and 0.001 , respectively), compared to those with a high MPV. The multivariate analysis demonstrated that a low MPV was an independent unfavorable prognostic factor for DFS and OS [hazard ratio $(\mathrm{HR})=1.713$; 95\% confidence interval $(\mathrm{CI}): 1.070-2.742, \mathrm{P}=0.025$; and $\mathrm{HR}=2.835$; 95\% CI: 1.304-6.163, $\mathrm{P}=0.009$, respectively)]. Therefore, we demonstrated that a low MPV predicted an unfavorable prognosis in patients with NSCLC following curative resection.
\end{abstract}

Correspondence to: Dr Cheng-Long Huang, Respiratory Disease Center, Tazuke Kofukai Medical Research Institute, Kitano Hospital, 2-4-20 Ohgimachi, Kita-ku, Osaka 530-8480, Japan

E-mail: chuang@kitano-hp.or.jp

Key words: lung cancer, mean platelet volume, overall survival, disease-free survival, curative resection, prognosis

\section{Introduction}

An increased mean platelet volume (MPV) is an early marker of platelet activation (1). Larger platelets may be more readily stimulated to release chemical mediators; therefore, larger platelets are recognized as being more reactive compared to smaller ones (2). MPV has been found to be increased in patients with various thromboembolic disorders $(3,4)$. Several studies assessed the prognostic significance of platelet count in patients with non-small-cell lung cancer (NSCLC) $(5,6)$. Thrombocytosis was noted to predict a poor overall survival (OS). Furthermore, a previous study demonstrated that a low MPV was significantly associated with an inferior OS in patients with advanced NSCLC (7). However, there has been no analysis of the prognostic effect of MPV on patients with resected NSCLC. The purpose of this study was to investigate the effect of MPV on survival in patients with completely resected NSCLC.

\section{Materials and methods}

Patient selection. We conducted a retrospective analysis of patients diagnosed with NSCLC who underwent surgery at the Tazuke Kofukai Medical Research Institute, Kitano Hospital, between January, 2007 and December,2011. All the patients met the following criteria: pathological confirmation of NSCLC; complete curative resection; no preoperative treatment; no microscopic residual tumor; no history of transplantation and/or immunosuppression; no evidence of infection, such as pneumonia, prior to surgery; no treatment for concomitant autoimmune diseases with immunosuppressive therapy; no history of hematological malignancy, including malignant lymphoma and leukemia; and availability of laboratory data and follow-up information.

Sample collection and staging. A peripheral venous blood sample was collected from each patient within a month prior to surgery. A blood test was performed using a fully automated blood cell counting system. Histological classification was performed according to the WHO guidelines (8). Disease staging was based on the 7th edition of the TNM classification of malignant tumors (9). Study approval was granted by the Ethics Committee of the Tazuke Kofukai Medical Research Institute, Kitano Hospital. 
Table I. Association between MPV and clinicopathological factors.

\begin{tabular}{lccr}
\hline & \multicolumn{2}{c}{ MPV } & \\
\cline { 2 - 3 } Variables & $<8.50 \mathrm{fl}(\mathrm{n}=176)$ & $\geq 8.50 \mathrm{fl}(\mathrm{n}=132)$ & P-value \\
\hline Age, years [median (range)] & $69(19-87)$ & $68(37-85)$ & 0.414 \\
Gender (male/female) & $98 / 78$ & $66 / 66$ & 0.357 \\
ECOG performance status (0/1/2) & $170 / 4 / 2$ & $127 / 4 / 1$ & 0.924 \\
Smoking history (never/ever) & $64 / 112$ & $58 / 74$ & 0.196 \\
Resected side (right/left) & $101 / 75$ & $79 / 53$ & 0.726 \\
Surgical procedure (pneumo/lob/seg) & $1 / 155 / 20$ & $0 / 110 / 22$ & 0.240 \\
Pathological stage (I/II/IIIA) & $129 / 18 / 29$ & $100 / 18 / 14$ & 0.265 \\
Tumor status (T1/T2/T3/T4) & $102 / 60 / 10 / 4$ & $81 / 43 / 6 / 2$ & 0.907 \\
Lymph node status (N0/N1/N2) & $140 / 9 / 27$ & $111 / 9 / 12$ & 0.221 \\
Histology (Ad/Sq/other) & $139 / 25 / 12$ & $103 / 25 / 4$ & 0.214 \\
Pleural invasion (present/absent) & $57 / 119$ & $29 / 103$ & 0.054 \\
Postoperative adjuvant chemotherapy & 42 & 22 & 0.156 \\
Recurrence of lung cancer & 40 & 24 & 0.395 \\
Cause of death (lung cancer/other/unknown) & $15 / 20 / 4$ & $5 / 3 / 0$ & 0.504 \\
\hline
\end{tabular}

MPV, mean platelet volume; ECOG, Eastern Cooperative Oncology Group; pneumo, pneumonectomy; lob, lobectomy or bilobectomy; seg, segmentectomy; Ad, adenocarcinoma; Sq, squamous cell carcinoma.

Clinicopathological characteristics. The following clinical characteristics were retrieved from the available clinical records: age, gender, Eastern Cooperative Oncology Group (ECOG) performance status, smoking history, pathological stage, pathological tumor status, pathological lymph node status, pleural invasion, peripheral platelet count and MPV.

Survival analysis. Disease-free survival (DFS) was measured from the date of surgery until the date of disease recurrence or death, or until the date the patient was last known to be disease-free. OS was measured from the date of surgery until the date of death from any cause or until the date on which the patient was last known to be alive. We estimated DFS and OS employing the Kaplan-Meier analysis (10). Differences between survival curves were tested for statistical significance using the two-tailed log-rank test. Univariate and multivariate prognostic analyses were performed for OS and DFS outcomes using the Cox proportional hazards model. Receiver operating characteristic (ROC) curve analysis was used to determine the optimal cut-off value for MPV; values with maximum joint sensitivity and specificity were selected. Categorical variables were compared using the $\chi^{2}$ test and continuous variables were compared using the t-test. All the statistical analyses were performed employing the R statistical software, version 2.13.1 (R Foundation for Statistical Computing, Vienna, Austria). All the $\mathrm{P}$-values are two-sided and $\mathrm{P}$-values $<0.05$ were considered to indicate statistically significant differences.

\section{Results}

Patients. Data from 373 patients diagnosed with NSCLC who underwent surgery at our hospital between January, 2007 and
December, 2011 were reviewed from the hospital's database and 65 patients were excluded due to preoperative treatment $(n=20)$, incomplete resection $(n=37)$ and history of hematological disorders $(n=8)$. Thus, 308 patients were finally included in this study. The clinicopathological characteristics of the patients are summarized in Table I. The patients included 164 men and 144 women, with a median age at the time of surgery of 69 years (range, 19-87 years). The median follow-up duration was 36.0 months (range, 1.0-77.4 months). A total of 64 patients received postoperative adjuvant chemotherapy and 64 patients experienced NSCLC recurrence. The 5-year DFS and OS rate of all 308 patients were $66.2 \%$ (pathological stage I, 76.5\%; pathological stage II, 39.6\%; and pathological stage IIIA, $33.5 \%$ ) and $80.5 \%$ (pathological stage I, $85.1 \%$; pathological stage II, $78.3 \%$; and pathological stage IIIA, $56.9 \%)$, respectively.

Optimal cut-off values for MPV and association between $M P V$ and clinicopathological factors. The ROC curve for MPV was used to determine the cut-off values. The area under the curve for MPV was 0.641 [95\% confidence interval (CI): 0.562-0.719]. An MPV of $8.50 \mathrm{fl}$ corresponded to the maximum joint sensitivity and specificity on the ROC curve ( $87.2 \%$ sensitivity and $44.1 \%$ specificity). The associations between MPV and the clinicopathological factors in this study population are shown in Table I. A low MPV was not found to be significantly associated with any other clinicopathological factor.

Evaluation of the prognostic effect of MPV. The Kaplan-Meier analysis was performed to determine whether MPV was associated with DFS and OS. The DFS was significantly shorter 

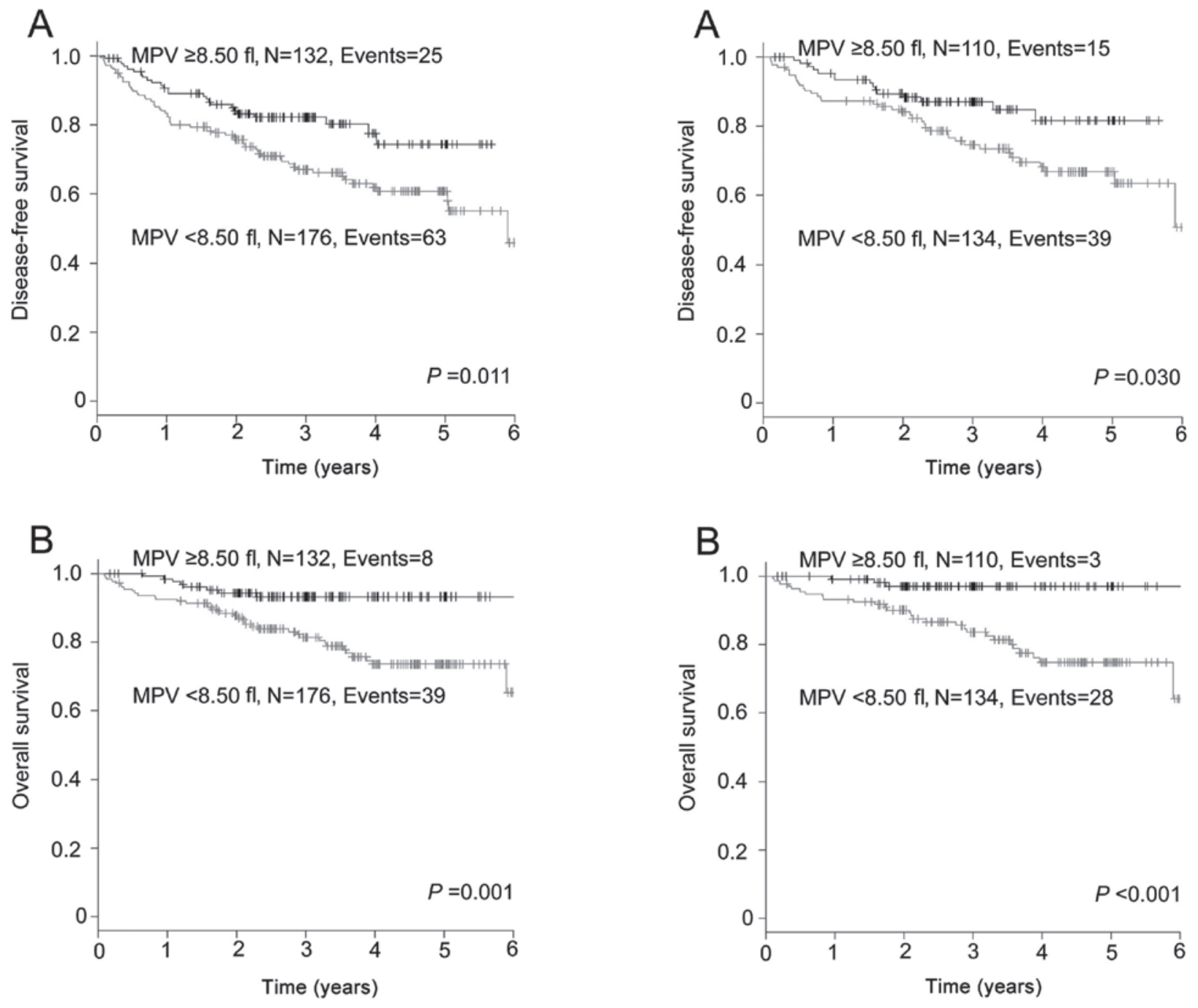

Figure 1. Kaplan-Meier analyses of (A) disease-free survival and (B) overall survival of all the patients included in this study stratified by mean platelet volume (MPV; $<8.50$ vs. $\geq 8.50 \mathrm{fl}$ ).

in the group with a MPV of $<8.50 \mathrm{fl}$ compared to that in the group with a MPV of $\geq 8.50 \mathrm{fl}$ ( $\mathrm{P}=0.011$; Fig. $1 \mathrm{~A})$. The 5-year DFS rate in the $<8.50 \mathrm{fl}$ and $\geq 8.50 \mathrm{fl}$ groups was 60.8 and $74.4 \%$, respectively. The OS in the group with a MPV of $<8.50 \mathrm{fl}$ was significantly inferior to that in the group with a MPV of $\geq 8.50 \mathrm{fl}$ ( $\mathrm{P}=0.001$; Fig. 1B). The 5 -year OS rate in the two groups was 73.6 and $93.3 \%$, respectively.

We also evaluated the effect of MPV on the prognosis of the 244 patients who did not receive adjuvant chemotherapy, employing the Kaplan-Meier analysis. The DFS of the group with a MPV of $<8.50 \mathrm{fl}$ was significantly shorter compared to that in the group with a MPV of $\geq 8.50 \mathrm{fl}$ ( $\mathrm{P}=0.030$; Fig. 2A). The 5 -year DFS rate in the $<8.50$ and $\geq 8.50 \mathrm{fl}$ groups was 66.8 and $81.6 \%$, respectively. The group with a MPV of $<8.50 \mathrm{fl}$ experienced a significantly inferior OS compared to that in the group with a MPV of $\geq 8.50 \mathrm{fl}$ ( $\mathrm{P}<0.001$; Fig. $2 \mathrm{~B})$. The 5 -year OS rate in the two groups was 74.8 and $97.0 \%$, respectively.

Univariate and multivariate analyses of factors associated with prognosis. The univariate analysis identified seven significant risk factors for DFS, namely age, gender, smoking history, tumor status, lymph node status, histology and

Figure 2. Kaplan-Meier analyses of (A) disease-free survival and (B) overall survival of the patients who did not receive adjuvant chemotherapy stratified by mean platelet volume (MPV; $<8.50 \mathrm{vs}$. $\geq 8.50 \mathrm{fl}$ ).

MPV (Table II). In the multivariate analysis, a low MPV was shown to be a statistically significant independent predictor of DFS, [hazard ratio (HR) $=1.713$; 95\% CI: 1.070-2.742, $\mathrm{P}=0.025]$. The other independent prognostic factors were age $(\mathrm{HR}=1.753$; $95 \% \mathrm{CI}: 1.127-2.727, \mathrm{P}=0.013)$, tumor status (HR=1.716; 95\% CI: 1.063-2.770, $\mathrm{P}=0.027)$ and lymph node status (HR=3.493; 95\% CI: 2.144-5.689, $\mathrm{P}<0.001)$.

As regards OS, the univariate analysis identified seven significant risk factors, namely age, gender, smoking history, tumor status, lymph node status, histology and MPV (Table III). The multivariate analysis identified low MPV as a statistically significant independent prognostic factor of OS (HR=2.835; 95\% CI: 1.304-6.163, $\mathrm{P}=0.009)$. The other independent prognostic factors were age $(\mathrm{HR}=4.466$; 95\% CI: $2.223-8.972, \mathrm{P}<0.001)$ and lymph node status (HR=3.654; 95\% CI: 1.881-7.098, $\mathrm{P}<0.001)$.

\section{Discussion}

In this study, we demonstrated that a low MPV was a poor prognostic factor in patients with NSCLC who received curative resection. A low MPV prior to the initiation of 
Table II. Prognostic effect of clinicopathological factors on disease-free survival in NSCLC.

\begin{tabular}{|c|c|c|c|c|c|c|}
\hline \multirow[b]{2}{*}{ Variables } & \multicolumn{3}{|c|}{ Univariate analysis } & \multicolumn{3}{|c|}{ Multivariate analysis } \\
\hline & HR & $95 \% \mathrm{CI}$ & P-value & HR & $95 \% \mathrm{CI}$ & P-value \\
\hline \multicolumn{7}{|l|}{ Age (years) } \\
\hline$\geq 70$ & 1.756 & $1.148-2.684$ & 0.009 & 1.753 & $1.127-2.727$ & 0.013 \\
\hline \multicolumn{7}{|l|}{ Gender } \\
\hline Male & 2.382 & $1.513-3.751$ & $<0.001$ & 1.789 & $0.954-3.354$ & 0.070 \\
\hline \multicolumn{7}{|c|}{ Smoking history } \\
\hline Ever & 2.001 & $1.258-3.184$ & 0.003 & 1.357 & $0.691-2.665$ & 0.375 \\
\hline \multicolumn{7}{|c|}{ Tumor status } \\
\hline $\mathrm{T} 2 / \mathrm{T} 3 / \mathrm{T} 4$ & 2.762 & $1.797-4.244$ & $<0.001$ & 1.716 & $1.063-2.770$ & 0.027 \\
\hline \multicolumn{7}{|c|}{ Lymph node status } \\
\hline $\mathrm{N} 1 / \mathrm{N} 2$ & 4.096 & $2.667-6.289$ & $<0.001$ & 3.493 & $2.144-5.689$ & $<0.001$ \\
\hline \multicolumn{7}{|l|}{ Histology } \\
\hline Non-Ad & 1.916 & $1.209-3.037$ & 0.006 & 0.773 & $0.445-1.343$ & 0.361 \\
\hline \multicolumn{7}{|l|}{ MPV } \\
\hline$\leq 8.50 \mathrm{fL}$ & 1.813 & $1.138-2.888$ & 0.012 & 1.713 & $1.070-2.742$ & 0.025 \\
\hline
\end{tabular}

NSCLC, non-small-cell lung cancer; HR, hazard ratio; 95\% CI, 95\% confidence interval; MPV, mean platelet volume; Ad, adenocarcinoma.

Table III. Prognostic effect of clinicopathological factors on overall survival in NSCLC.

\begin{tabular}{|c|c|c|c|c|c|c|}
\hline \multirow[b]{2}{*}{ Variables } & \multicolumn{3}{|c|}{ Univariate analysis } & \multicolumn{3}{|c|}{ Multivariate analysis } \\
\hline & HR & $95 \% \mathrm{CI}$ & P-value & $\mathrm{HR}$ & $95 \% \mathrm{CI}$ & P-value \\
\hline \multicolumn{7}{|l|}{ Age (years) } \\
\hline$\geq 70$ & 3.800 & $1.971-7.329$ & $<0.001$ & 4.466 & $2.223-8.972$ & $<0.001$ \\
\hline \multicolumn{7}{|l|}{ Gender } \\
\hline Male & 2.820 & $1.462-5.438$ & 0.002 & 2.197 & $0.822-5.874$ & 0.117 \\
\hline \multicolumn{7}{|c|}{ Smoking history } \\
\hline Ever & 2.419 & $1.231-4.755$ & 0.010 & 1.376 & $0.484-3.918$ & 0.550 \\
\hline \multicolumn{7}{|c|}{ Tumor status } \\
\hline $\mathrm{T} 2 / \mathrm{T} 3 / \mathrm{T} 4$ & 2.110 & $1.181-3.768$ & 0.012 & 1.325 & $0.703-2.498$ & 0.385 \\
\hline \multicolumn{7}{|c|}{ Lymph node status } \\
\hline $\mathrm{N} 1 / \mathrm{N} 2$ & 3.388 & $1.891-6.069$ & $<0.001$ & 3.654 & $1.881-7.098$ & $<0.001$ \\
\hline \multicolumn{7}{|l|}{ Histology } \\
\hline Non-Ad & 1.881 & $1.003-3.528$ & 0.049 & 0.649 & $0.307-1.374$ & 0.259 \\
\hline \multicolumn{7}{|l|}{ MPV } \\
\hline$\leq 8.50 \mathrm{fl}$ & 3.303 & $1.539-7.086$ & 0.002 & 2.835 & $1.304-6.163$ & 0.009 \\
\hline
\end{tabular}

NSCLC, non-small-cell lung cancer; HR, hazard ratio; 95\% CI, 95\% confidence interval; MPV, mean platelet volume; Ad, adenocarcinoma.

treatment was reported to predict a poor prognosis in advanced NSCLC (7). To the best of our knowledge, this is the first study to demonstrate the prognostic effect of MPV on DFS and OS in patients with completely resected NSCLC.

MPV has been considered to reflect platelet activity, as it was shown to be associated with platelet aggregation (11), thromboxane B2 release (12) and increased expression of the platelet adhesion molecule glycoprotein IIb/IIIa (13). Several clinical studies reported that an elevated MPV is associated with thromboembolic diseases, such as myocardial infarction (3) or stroke (4). Recently, the association between MPV and various types of cancer has attracted significant attention. A significant decrease in MPV was demonstrated in various cancer patients with metastasis to the bone marrow compared 
to control subjects (14). In addition, MPV was found to be decreased in patients with advanced NSCLC (9).

The association between coagulation and cancer may be key to explaining the decrease in MPV. A recent report demonstrated that lung cancer cell-derived microparticles bearing $\mathrm{P}$-selectin and tissue factor activate the circulating platelets, leading to thrombus formation (15). Furthermore, tumor cells release various soluble proinflammatory (i.e., tumor necrosis factor- $\alpha$ and interleukin-1 $\beta$ ) and proangiogenic factors (i.e., vascular endothelial growth factor and basic fibroblast growth factor) (16), which may stimulate the prothrombotic properties of vascular cells. The tendency of larger platelets to react to stimuli causes selective consumption of larger platelets, with a resulting decrease in the MPV of circulating platelets. Mutlu et al (17) analyzed MPV levels in patients with various cancers and found a significant decrease in MPV levels at the time of the thrombotic events compared to those at diagnosis. In 241 patients $(78.2 \%)$ of the population included in that study, the MPV was below the normal limit, which may support the hypothesis that MPV decreases in patients with NSCLC. Further studies on the association between MPV and cancer-related thrombosis are required.

The effect of platelets on the survival of patients with cancer has been extensively investigated. Platelets have been suggested to play an important role in cancer progression and metastasis (18). A previous study demonstrated that preliminarily activated platelets have tumor-promoting properties (19). Although the number of studies that have investigated the biological association between MPV and cancer progression is limited, recent clinical reports demonstrated the negative effect of a low MPV on the prognosis of cancer patients. Riedl et al (20) evaluated the data of 1,544 patients with various types of cancer and found that high MPV values were associated not only with a decreased risk of venous thromboembolism, but also with an improved patient survival. As for advanced NSCLC, a low MPV prior to the treatment was shown to predict a poor prognosis (7). The present study also demonstrated the negative effect of a low MPV on the DFS and OS of NSCLC patients who received complete resection, even subtracting the effects of adjuvant chemotherapy, which supports the findings of previous studies. However, these results should be verified in a prospective study with a larger sample size.

In this study, we demonstrated that a low MPV prior to surgery was an independent unfavorable prognostic factor in patients who underwent complete resection of NSCLC. MPV, available in a routine complete blood count examination, may represent one of the easiest measurements to be used as a prognostic marker independent of tumor status or lymph node status in NSCLC. Therefore, preoperative MPV may be a useful tool for selecting optimal treatment strategies, including adjuvant chemotherapy. The limitations of the present study include its retrospective design and relatively short follow-up duration. Further investigations are required to elucidate the precise mechanisms through which circulating platelets affect the prognosis of patients with NSCLC.

\section{References}

1. Thompson CB and Jakubowski JA: The pathophysiology and clinical relevance of platelet heterogeneity. Blood 72: 1-8, 1988.

2. Thompson CB, Jakubowski JA, Quinn PG, et al: Platelet size and age determine platelet function independently. Blood 63: 1372-1375, 1984.

3. Martin JF, Bath PM and Burr ML: Influence of platelet size on outcome after myocardial infarction. Lancet 338: 1409-1411, 1991.

4. Tohgi H, Suzuki H, Tamura K, et al: Platelet volume, aggregation, and adenosine triphosphate release in cerebral thrombosis. Stroke 22: 17-21, 1991.

5. Aoe K, Hiraki A, Ueoka H, et al: Thrombocytosis as a useful prognostic indicator in patients with lung cancer. Respiration 71: $170-173,2004$

6. Maráz A, Furák J, Varga Z, et al: Thrombocytosis has a negative prognostic value in lung cancer. Anticancer Res 33: 1725-1730, 2013.

7. Inagaki N, Kibata K, Tamaki T, et al: Prognostic impact of the mean platelet volume/platelet count ratio in terms of survival in advanced non-small cell lung cancer. Lung Cancer 83: 97-101, 2014.

8. World Health Organization: Tumours of the lung. In: Tumours of the Lung, Pleura, Thymus and Heart. Travis WD, Bramvilla E, Muller-Hermelink HK and Harris CC (eds). International Agency for Research on Cancer, Lyon, p10, 2004.

9. Sobin LH, Gospodarowicz MK and Wittekind C: International Union Against Cancer (UICC): TNM classification of malignant tumours. 7th edition. John Willkey and Sons, Ltd., UK, 2010.

10. Kaplan E and Meier P: Nonparametric estimation from incomplete observations. J Am Stat Assoc 53: 457-481, 1958.

11. Thompson CB, Eaton KA, Princiotta SM, et al: Size dependent platelet subpopulations: relationship of platelet volume to ultrastructure, enzymatic activity, and function. Br J Haematol 50: 509-519, 1982 .

12. Jakubowski JA, Thompson CB, Vaillancourt R, et al: Arachidonic acid metabolism by platelets of differing size. Br J Haematol 53: 503-511, 1983.

13. Giles H, Smith RE and Martin JF: Platelet glycoprotein IIb-IIIa and size are increased in acute myocardial infarction. Eur J Clin Invest 24: 69-72, 1994

14. Aksoy S, Kilickap S, Hayran M, et al: Platelet size has diagnostic predictive value for bone marrow metastasis in patients with solid tumors. Int J Lab Hematol 30: 214-219, 2008.

15. Thomas GM, Panicot-Dubois L, Lacroix R, et al: Cancer cell-derived microparticles bearing P-selectin glycoprotein ligand 1 accelerate thrombus formation in vivo. J Exp Med 206: 1913-1927, 2009.

16. Falanga A, Panova-Noeva $M$ and Russo L: Procoagulant mechanisms in tumour cells. Best Pract Res Clin Haematol 22: 49-60, 2009.

17. Mutlu H, Artis TA, Erden A and Akca Z: Alteration in mean platelet volume and platicrit values in patients with cancer that developed thrombosis. Clin Appl Thromb Hemost 19: 331-333, 2013.

18. Nash GF, Turner LF, Scully MF, et al: Platelets and cancer. Lancet Oncol 3: 425-430, 2002.

19. Zhang W, Dang S, Hong T, et al: A humanized single-chain antibody against beta 3 integrin inhibits pulmonary metastasis by preferentially fragmenting activated platelets in the tumor microenvironment. Blood 120: 2889-2898, 2012.

20. Riedl J, Kaider A, Reitter EM, et al: Association of mean platelet volume with risk of venous thromboembolism and mortality in patients with cancer. Results from the Vienna Cancer and Thrombosis Study (CATS). Thromb Haemost 111: 670-678, 2014. 